\title{
Fixed Points of Occasionally Weakly Compatible Maps Satisfying General Contractive Conditions of Integral Type
}

\author{
HAKIMA BOUHADJERA
}

\begin{abstract}
In this paper, two common fixed point theorems for four occasionally weakly compatible maps satisfying a contractive condition of integral type are obtained. Our results improve some results especially Theorem 2.1 of [3] and Theorem 1 of [1].
\end{abstract}

\section{Introduction ANd Preliminaries}

In 1982 Sessa [6] generalized the concept of commuting maps by calling self-maps $f$ and $g$ of a metric space $(\mathcal{X}, d)$ a weakly commuting pair if and only if for all $x \in \mathcal{X}$ :

$$
d(f g x, g f x) \leq d(g x, f x) .
$$

In 1986 Jungck [4] made a generalization of concept of weakly commutativity called compatibility. $f$ and $g$ are compatible if:

$$
\lim _{n \rightarrow \infty} d\left(f g x_{n}, g f x_{n}\right)=0
$$

whenever $\left\{x_{n}\right\}$ is a sequence in $\mathcal{X}$ such that $\lim _{n \rightarrow \infty} f x_{n}=\lim _{n \rightarrow \infty} g x_{n}=t$ for some $t \in \mathcal{X}$.

Further, the same author [5] defined the concept of weak compatibility which generalized the notion of compatibility. $f$ and $g$ above are said to be weakly compatible if they commute at their coincidence points.

Recently in 2008, Al-Thagafi with Shahzad [2] gave a proper generalization of weakly compatible maps by introducing the concept of occasionally weakly compatible maps (shortly (owc)). Two self-maps $f$ and $g$ of a set $\mathcal{X}$ are owc if and only if there is a point $t \in \mathcal{X}$ which is a coincidence point of $f$ and $g$ at which $f$ and $g$ commute.

Before giving our main results, recall that a symmetric on a set $\mathcal{X}$ is a function $d: \mathcal{X} \times \mathcal{X} \rightarrow[0, \infty)$ satisfying the following conditions:

(1) $d(x, y)=0$, if and only if $x=y$ for $x, y \in \mathcal{X}$,

(2) $d(x, y)=d(y, x)$, for all $x, y \in \mathcal{X}$.

2000 Mathematics Subject Classification. Primary: 47H10, 54H25.

Key words and phrases. Weakly compatible maps, occasionally weakly compatible maps, contractive condition, integral type, common fixed point theorems. 


\section{Main Results}

Now we give our main results. We begin by citing and proving our first theorem.

Theorem 2.1. Let $d$ be a symmetric for $\mathcal{X}$. Let $h, k, f$ and $g$ be self-maps of $\mathcal{X}$ such that for all $x, y$ in $\mathcal{X}$, there exists a function $\psi: \mathbb{R}_{+} \rightarrow \mathbb{R}_{+}$, $\psi(0)=0, \psi(t)<t$ for $t>0$, and

$$
\int_{0}^{d(f x, g y)} \varphi(t) \mathrm{d} t \leq \psi\left(\int_{0}^{M(x, y)} \varphi(t) \mathrm{d} t\right),
$$

where $\varphi: \mathbb{R}_{+} \rightarrow \mathbb{R}_{+}$is a Lebesgue-integrable map which is summable nonegative such that $\int_{0}^{\varepsilon} \varphi(t) \mathrm{d} t>0$ for each $\varepsilon>0$,

$M(x, y)=\max \left\{d(h x, k y), d(f x, h x), d(g y, k y) \frac{1}{2}(d(f x, k y)+d(g y, h x))\right\}$.

If the pairs $\{f, h\}$ and $\{g, k\}$ are owc, then $h, k, f$ and $g$ have a unique common fixed point in $\mathcal{X}$.

Proof. By hypothesis, there are two points $u$ and $v$ in $\mathcal{X}$ such that $f u=h u$ and $f h u=h f u, g v=k v$ and $g k v=k g v$.

We claim that $f u=g v$. If not, from (1):

$$
\begin{aligned}
& \int_{0}^{d(f u, g v)} \varphi(t) \mathrm{d} t \leq \psi\left(\int_{0}^{M(u, v)} \varphi(t) \mathrm{d} t\right)= \\
& =\psi\left(\int_{0}^{\max \left\{d(h u, k v), d(f u, h u), d(g v, k v), \frac{1}{2}(d(f u, k v)+d(g v, h u))\right\}}\right)= \\
& =\psi\left(\int_{0}^{d(f u, g v)} \varphi(t) \mathrm{d} t\right)<\int_{0}^{d(f u, g v)} \varphi(t) \mathrm{d} t,
\end{aligned}
$$

a contradiction. Therefore $f u=h u=g v=k v$.

Suppose that $f^{2} u \neq f u$, then inequality (1) gives:

$$
\begin{aligned}
& \int_{0}^{d\left(f^{2} u, f u\right)} \varphi(t) \mathrm{d} t=\int_{0}^{d(f f u, g v)} \varphi(t) \mathrm{d} t \leq \\
& \quad \leq \psi\left(\int_{0}^{M(f u, v)} \varphi(t) \mathrm{d} t\right)= \\
& \quad=\psi\left(\int_{0}^{\max \left\{d(h f u, k v), d\left(f^{2} u, h f u\right), d(g v, k v), \frac{1}{2}\left(d\left(f^{2} u, k v\right)+d(g v, h f u)\right)\right\}} \varphi(t) \mathrm{d} t\right)= \\
& \quad=\psi\left(\int_{0}^{d\left(f^{2} u, f u\right)} \varphi(t) \mathrm{d} t\right)<\int_{0}^{d\left(f^{2} u, f u\right)} \varphi(t) \mathrm{d} t,
\end{aligned}
$$


which is a contradiction. Hence, $f^{2} u=f u=h f u$.

Similarly, $g^{2} v=g v=k g v$, and $f u=h u=g v=k v$ is a common fixed point of $f, h, g$ and $k$.

The uniqueness of the common fixed point follows easily from condition (1).

If we let $f=g$ and $k=h$ in Theorem 2.1, we get the following corollary.

Corollary 2.1. Let $d$ be a symmetric for $\mathcal{X}$ and let $f$ and $h$ be self-maps of $\mathcal{X}$ such that for all $x, y$ in $\mathcal{X}$, there is a function $\psi: \mathbb{R}_{+} \rightarrow \mathbb{R}_{+}, \psi(0)=0$, $\psi(t)<t$ for $t>0$, and

$$
\begin{aligned}
& \int_{0}^{d(f x, f y)} \varphi(t) \mathrm{d} t \leq \\
& \quad \leq \psi\left(\int_{0}^{\max \left\{d(h x, h y), d(f x, h x), d(f y, h y), \frac{1}{2}(d(f x, h y)+d(f y, h x))\right\}} \varphi(t) \mathrm{d} t\right),
\end{aligned}
$$

where $\varphi$ is as in Theorem 2.1. If $f$ and $h$ are owc, then $f$ and $h$ have a unique common fixed point in $\mathcal{X}$.

Now, if we put $k=h$ in Theorem 2.1, we get the following result.

Corollary 2.2. Let $d$ be a symmetric for $\mathcal{X}$. Let $h, f$ and $g$ be three selfmaps of $\mathcal{X}$ such that for all $x, y$ in $\mathcal{X}$, there is a function $\psi: \mathbb{R}_{+} \rightarrow \mathbb{R}_{+}$, $\psi(0)=0, \psi(t)<t$ for $t>0$, and

$$
\begin{aligned}
& \int_{0}^{d(f x, g y)} \varphi(t) \mathrm{d} t \leq \\
& \quad \leq \psi\left(\int_{0}^{\max \left\{d(h x, h y), d(f x, h x), d(g y, h y), \frac{1}{2}(d(f x, h y)+d(g y, h x))\right\}} \varphi(t) \mathrm{d} t\right),
\end{aligned}
$$

where $\varphi$ is as in Theorem 2.1. If $f$ and $h$, as well as $g$ and $h$, are owc, then $f, g$ and $h$ have a unique common fixed point in $\mathcal{X}$.

Theorem 2.2. Let $d$ be a symmetric for the set $\mathcal{X}$ and let $h, k, f$ and $g$ be self-maps of $\mathcal{X}$ such that

$$
\begin{aligned}
& \int_{0}^{d(h x, k y)} \varphi(t) \mathrm{d} t \leq \\
& \quad \leq \psi\left(\int_{0}^{\max \{d(f x, g y), d(f x, k y), d(k y, g y)\}} \varphi(t) \mathrm{d} t\right),
\end{aligned}
$$

for all $x, y$ in $\mathcal{X}$, where $\varphi$ and $\psi$ are as in Theorem 2.1. If the pairs $\{h, f\}$ and $\{k, g\}$ are owc, then $h, k, f$, and $g$ have a unique common fixed point in $\mathcal{X}$. 
Proof. Existence. Since the pairs $\{h, f\}$ and $\{k, g\}$ are owc, then there are two elements $u, v$ in $\mathcal{X}$ such that $h u=f u$ and $h f u=f h u, k v=g v$ and $k g v=g k v$. First, we prove that $h u=k v$. Suppose not, then, by using inequality (2) we get:

$$
\begin{aligned}
& \int_{0}^{d(h u, k v)} \varphi(t) \mathrm{d} t \leq \\
& \quad \leq \psi\left(\int_{0}^{\max \{d(f u, g v), d(f u, k v), d(k v, g v),\}} \varphi(t) \mathrm{d} t\right)= \\
& \quad=\psi\left(\int_{0}^{d(h u, k v)} \varphi(t) \mathrm{d} t\right)<\int_{0}^{d(h u, k v)} \varphi(t) \mathrm{d} t,
\end{aligned}
$$

which is a contradiction. Therefore $f u=h u=k v=g v$

Now, we claim that $h h u=f h u=h u$. If not, then the use of condition (2) gives:

$$
\begin{aligned}
& \int_{0}^{d\left(h^{2} u, h u\right)} \varphi(t) \mathrm{d} t=\int_{0}^{d(h h u, k v)} \varphi(t) \mathrm{d} t \leq \\
& \quad \leq \psi\left(\int_{0}^{\max \{d(f h u, g v), d(f h u, k v), d(k v, g v),\}} \varphi(t) \mathrm{d} t\right)= \\
& \quad=\psi\left(\int_{0}^{d\left(h^{2} u, h u\right)} \varphi(t) \mathrm{d} t\right)<\int_{0}^{d\left(h^{2} u, h u\right)} \varphi(t) \mathrm{d} t,
\end{aligned}
$$

a contradiction. Hence $h^{2} u=f h u=h u$.

Similarly, $k^{2} v=g k v=k v$, and $h u=k v=z$ is a common fixed point of both $h, k, f$ and $g$.

Uniqueness. Suppose that there are two distinct points $z, z^{\prime}$ in $\mathcal{X}$, then by (2) we have the contradiction:

$$
\begin{aligned}
& \int_{0}^{d\left(z, z^{\prime}\right)} \varphi(t) \mathrm{d} t=\int_{0}^{d\left(h z, k z^{\prime}\right)} \varphi(t) \mathrm{d} t \leq \\
& \quad \leq \psi\left(\int_{0}^{\max \left\{d\left(f z, g z^{\prime}\right), d\left(f z, k z^{\prime}\right), d\left(k z^{\prime}, g z^{\prime}\right),\right\}} \varphi(t) \mathrm{d} t\right)= \\
& \quad=\psi\left(\int_{0}^{d\left(z, z^{\prime}\right)} \varphi(t) \mathrm{d} t\right)<\int_{0}^{d\left(z, z^{\prime}\right)} \varphi(t) \mathrm{d} t .
\end{aligned}
$$

The proof is completed. 
Corollary 2.3. Let $d$ be a symmetric for $\mathcal{X}$ and let $h, k$ be two owc self-maps of $\mathcal{X}$ such that:

$$
\begin{aligned}
& \int_{0}^{d(h x, h y)} \varphi(t) \mathrm{d} t \leq \\
& \quad \leq \psi\left(\int_{0}^{\max \{d(k x, k y), d(k x, h y), d(h y, k y),\}} \varphi(t) \mathrm{d} t\right)
\end{aligned}
$$

for all $x, y$ in $\mathcal{X}$, where $\varphi$ and $\psi$ are as in Theorem 2.1, then $h$ and $k$ have a unique common fixed point in $\mathcal{X}$.

Corollary 2.4. Let $d$ be a symmetric for $\mathcal{X}$ and let $h, k$ and $f$ be self-maps of $\mathcal{X}$ satisfying the following inequality:

$$
\begin{aligned}
& \int_{0}^{d(h x, k y)} \varphi(t) \mathrm{d} t \leq \\
& \quad \leq \psi\left(\int_{0}^{\max \{d(f x, f y), d(f x, k y), d(k y, f y),\}} \varphi(t) \mathrm{d} t\right)
\end{aligned}
$$

for all $x, y$ in $\mathcal{X}$, where $\varphi$ and $\psi$ are as in Theorem 2.1. If pairs $\{h, f\}$ and $\{k, f\}$ are owc, then $h, k$ and $f$ have a unique common fixed point in $\mathcal{X}$.

\section{REFERENCES}

[1] A. Aliouche, A common fixed point theorem for weakly compatible mappings in symmetric spaces satisfying a contractive condition of integral type, J. Math. Anal. Appl., 322 (2006), No. 2, 796-802.

[2] M.A. Al-Thagafi, N. Shahzad, Generalized I-nonexpansive selfmaps and invariant approximations, Acta Math. Sin. (Engl. Ser.) 24 (2008), No. 5, 867-876.

[3] I. Altun, D. Türkoğlu, B.E. Rhoades, Fixed points of weakly compatible maps satisfying a general contractive condition of integral type, Fixed Point Theory Appl. 2007, Art. ID 17301, 9 pp.

[4] G. Jungck, Compatible mappings and common fixed points, Internat. J. Math. Math. Sci., 9 (1986), No. 4, 771-779.

[5] G. Jungck, Common fixed points for noncontinuous nonself maps on non-metric spaces, Far East J. Math. Sci., 4 (1996), No. 2, 199-215.

[6] S. Sessa, On a weak commutativity condition of mappings in fixed point considerations, Publ. Inst. Math. (Beograd) (N.S.), 32(46) (1982), 149-153.

HaKima Bouhadjera

Laboratoire de Mathématiques Appliquées UNIVERSité BADJi MOKHTAR

B.P. 12, 23000, AnnabA

Algérie

E-mail address: b_hakima2000@yahoo.fr 
